\title{
Effects of heat treatment on the magnetic properties of melt-spun Nd6Pr1Fe76B12Ti4C1Co3 nanocomposite ribbons
}

\begin{abstract}
In this study, the effects of different annealing temperatures on the structure and magnetic properties of $\mathrm{Nd}-\mathrm{Fe}-\mathrm{B}$ nanocomposite permanent magnetic alloys with nominal composition of Nd6Pr1Fe76B12Ti4C1Co3 have been investigated. Melt Spinning (one of the most predominant ribbon synthesize methods) with constant wheel speed of $V=25 \mathrm{~m} / \mathrm{s}$ was employed to produce ribbons. As-spun ribbons were examined by using xray diffractometer (XRD) with $\mathrm{Cu}-\mathrm{K} U$ radiation and differential scanning calorimetry (DSC). The ribbons were annealed at different temperatures in order to extract the best magnetic properties. The XRD and electron microscopy technique results confirm that grains are in the size of less than 50 $\mathrm{nm}$. In addition, optimum magnetic properties were obtained at $720^{\circ} \mathrm{C}$ annealed temperature.
\end{abstract}

Keyword: Heat treatment; Melt-spun; Nanocomposite; Nd-Fe-B 